\title{
CHARACTERIZATION OF TWO-PHASE FLOWS USING FRACTAL ANALYSIS OF LOCAL TEMPERATURE FLUCTUATIONS
}

\author{
R. KOZMA', H. KOK ${ }^{2}$, M. SAKUMA', D. D. DJAINAL ${ }^{1}$, M. KITAMURA' \\ 'Department of Nuclear Engineering, Tohoku University Aramaki-Aza, Aoba, Sendai 980-77, Japan \\ ${ }^{2}$ Interfaculty Reactor Institute, Delft University of Technology Mekelweg 15, 2629 JB Delft,
} The Netherlands

(Received September 1995; in revised form May 1996)

\begin{abstract}
This work deals with the characterization of two-phase flows based on fractal techniques in order to develop objective flow regime indicators. Fractal analysis has been used previously for studying turbulent flows and it is a natural way of describing highly irregular fluctuations in two-phase flows. The fractal dimension of measured time series have been evaluated by Higuchi's method. It is shown that the error of the linear fit of the fractal dimension is a sensitive indicator of the changes in the flow regime, while the fractal dimension value itself is less suitable for flow regime identification. The developed method has been applied to the evaluation of two-phase flow experiments at the SIDAS boiling loop at Delft University of Technology, the Netherlands. Copyright (C) 1996 Elsevier Science Ltd.
\end{abstract}

Key Words: two-phase flow, flow regimes, subcooled boiling, bulk boiling, fractal dimension, temperature fluctuation

\section{INTRODUCTION}

Two-phase flow regimes have been studied by a number of authors to obtain objective criteria for the identification of various flow patterns. The applied methods include analysis of power spectral densities (PSDs), moments of probability density function (pdf) of detector signals and various pattern recognition techniques; see, e.g. Vince \& Lahey (1982), Dukler \& Taitel (1986), and Baba et al. (1991). Practical implementation of these methods requires knowledge on certain parameters of the two-phase flow, e.g. coolant and vapor phase velocities, which cannot be easily measured in some experimental arrangements. Therefore, further elaboration of techniques of two-phase flow regime identification in boiling coolant is of large practical importance.

Statistical fluctuations of two-phase flow parameters contain valuable information on the thermal-hydraulic state of the coolant. The chaotic character of these fluctuations can be analysed by evaluating various dynamic variables such as fractal dimension, Lyapunov exponent, Poincaré maps, etc. Following the introduction of self-similarity measures by Mandelbrot (1982), successful attempts have been made to apply fractal analysis to monitor two-phase flows; see Saether $e t$ al. (1990) and Franca et al. (1991). In the present work, two-phase flow regimes are monitored by analysing fractal features of thermal hydraulic processes in a vertical boiling channel. Preliminary results of this study are reported by Djainal et al. (1995). In the analysis, a novel fractal dimension evaluation method is used which was introduced by Higuchi (1988).

In the first part of the paper the theoretical background of fractal dimension estimation in time series is summarized. This is followed by a brief description of the experimental set-up and measurement conditions. In the second part of the paper fractal characteristics of signals of thermocouples located at various axial locations in a coolant channel are analysed. We use conventional statistical methods, like Fourier-spectrum evaluation and momentum analysis, together with fractal techniques. It has been found that the measured signals had bi-fractal properties, i.e. the fractal dimension has a dispersion regarding small and large wave numbers, respectively. It is also shown that the error of the linear fit of the fractal dimension value related to the bi-fractal feature of the measured signals is a sensitive indicator of the various flow regimes. 


\section{METHOD}

A detailed description of the existing methods of fractal dimension evaluation and also discussion of the validity of the different approaches is given by Schepers et al. (1992). A typical method is based on box-counting, i.e. dividing the data into elementary boxes and counting the number of boxes which contain data points as the function of the box size; Mandelbrot (1982). In the present work, the algorithm elaborated by Higuchi (1988) is used. According to Higuchi's method, the length of graph of the time series is calculated for different time lags $k$. The average partial length of the curve, $\langle L(k)\rangle$ is calculated for a given time series $X(j), j=1,2, \ldots, N$ and fixed $k$ as follows:

$$
\langle L(k)\rangle=\frac{1}{k} \sum_{m=1}^{k} \frac{N-1}{k} \sum_{i=1}^{[(N-m) ; k]} \frac{|X(m+i k)-X(m+(i-1) k)|}{k[(N-m) / k]} .
$$

Here $[(N-m) / k]$ stands for the integer part of $(N-m) / k$. It is easy to see that [1] indeed gives a normalized length by summing up the absolute values of the step-by-step changes along the curve of the time series. The mean length of the curve $\langle L(k)\rangle$ is calculated over a range of $k$ values. If the original signal is fractal, or if it reveals fractal characteristics over a fixed range of $k$, the following relationship holds:

$$
\langle L(k)\rangle \propto k^{-D},
$$

where $D$ is called the fractal dimension of the signal. Fractal dimension $D$ can be estimated through the slope of the $\log (k)$ vs $\log \langle L(k)\rangle$ plot. In order to estimate the magnitude and the error of the fractal dimension based on the slope of the log-log plot, the least squares method is used. By plotting the curve length values against the time scales on a $\log -\log$ axis, the data of the fractal time series fall on a straight line with slope $-D$.

The error of the estimated fractal dimension consists of two main components: the statistical uncertainty of the measurement data and the error caused by the deviation of the $\log (k)$ vs $\log (\langle L(k)\rangle)$ curve from the strict linear behavior. This deviation is characterized by the $\chi^{2}$ value of the linear fit and it is called the 'fractalness' of the investigated time series; see Sakuma $e t$ al. (1996). In the present work, we use a simple error measure based on the standard deviation of the linear fit of the fractal curve. This measure is proved to be very useful in flow regime identification problems as it is shown in the forthcoming discussions.

\section{EXPERIMENTAL}

\subsection{Description of the boiling loop SIDAS}

The fractal analysis method introduced above has been applied to the evaluation of measurement data obtained at the SIDAS thermal-hydraulic set-up at the Interfaculty Reactor Institute, Delft University of Technology, Delft, the Netherlands. SIDAS is a freon loop which is designed to simulate boiling effects in a nuclear reactor; for details, see, Graaf et al. (1994). The electrically heated section of SIDAS consists of 36 fuel rods arranged in a $6 \times 6$ lattice. The heated length of a fuel rod is $88.2 \mathrm{~cm}$. The axial distribution of the heat flux density in each fuel rod approximates a cosine shape with a maximum at medium height of the rods. By changing the heating power and the primary and feedwater flow rates, experiments with stationary and transient boiling conditions were performed at SIDAS. A schematic view of SIDAS is given in figure 1, including the heated assembly, riser, and recirculation loop sections.

In figure 1 , the locations of thermocouples $(T)$, pressure transducers $(P)$ and flowmeters $(F)$ are indicated, the signals of which were sampled and recorded during the experiments. In addition, a movable gamma densitometer was used for void fraction measurements. Viewing ports were built into the channel wall at four axial locations, allowing a visual inspection of the boiling process. In the present paper, signals of thermocouples located at elevation $2,31,51$, and $71 \mathrm{~cm}$ above the lower plenum of the bundle, are analysed in detail. The applied chromel-alumel thermocouples have a diameter of $0.5 \mathrm{~mm}$. The thermocouples are located in the coolant and have a relatively fast response time and a working frequency range up to, at least, $15-20 \mathrm{~Hz}$.

The gamma densitometer consists of an Am-241 source and a NaI detector. Both the source and 
the detector are collimated, so a narrow beam passes through the coolant channel. By measuring the attenuation of the gamma beam, the density of the coolant between the source and the detector is determined. This allows us to evaluate the chordal average void fraction along the beam. The applied procedure has been extended to void fraction determination in the case of a spatially heterogeneous void profile by conducting a series of measurements near the wall and in the central locations of the coolant channel.

\subsection{Parameters of the experiments}

In the experiments analysed in this paper, the coolant mass flow rate was constant while the heating power was reduced from 40 to $35 \mathrm{~kW}$. The pressure was kept constant at the value of $(11.1 \pm 0.05)$ bar. The observed pressure fluctuations around the mean value are due to hydraulic processes in the loop. The corresponding saturation temperature of the freon is between 46.2 and $45.8^{\circ} \mathrm{C}$.

On the one hand, there was single-phase coolant at the position of the lowest thermocouple in each experiment. In the upper part of the assembly, on the other hand, various types of boiling occurred. Results by gamma void measurements at axial locations 31,51 , and $71 \mathrm{~cm}$ are depicted in figure 2. Measurements have been compared with the results of thermal-hydraulic calculations

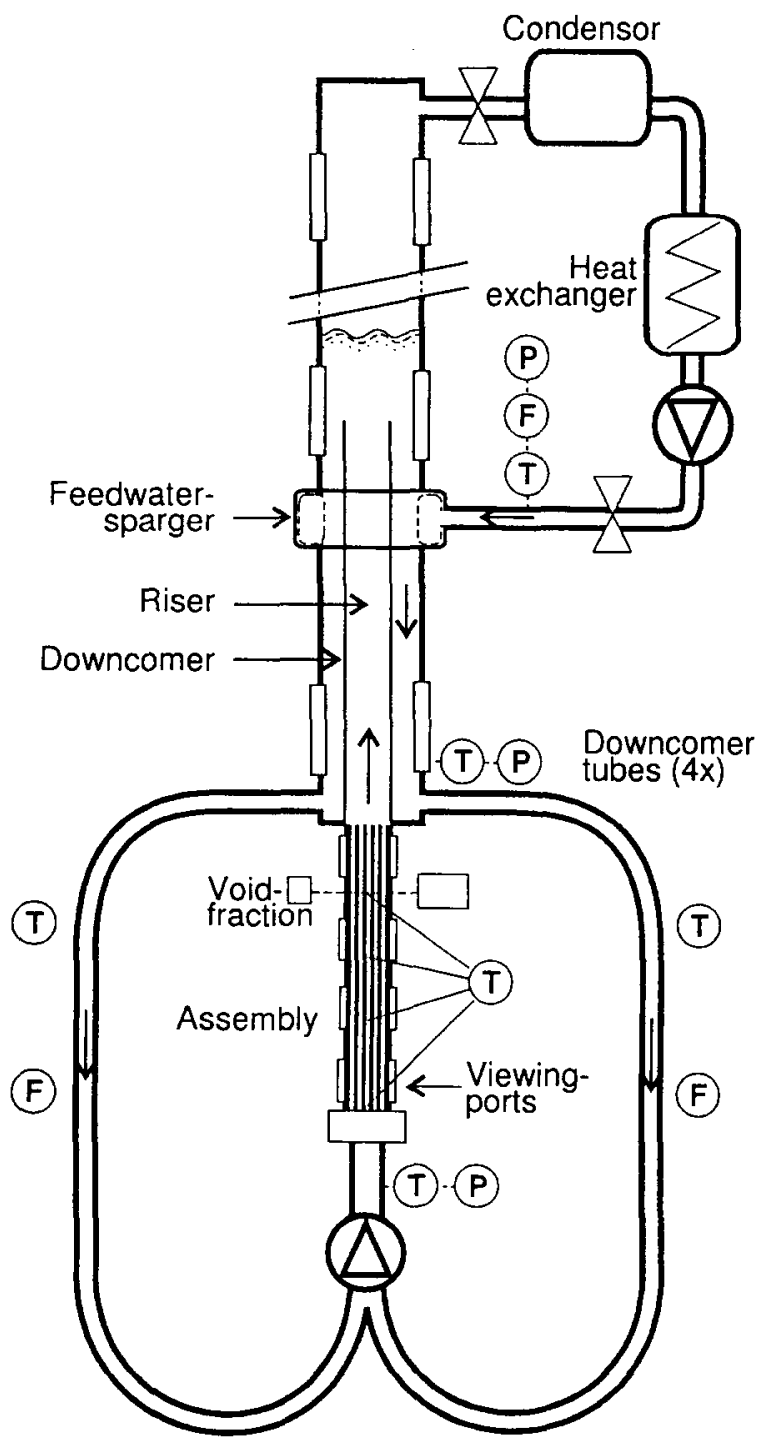

Figure 1. Schematic of the experimental set-up SIDAS. 


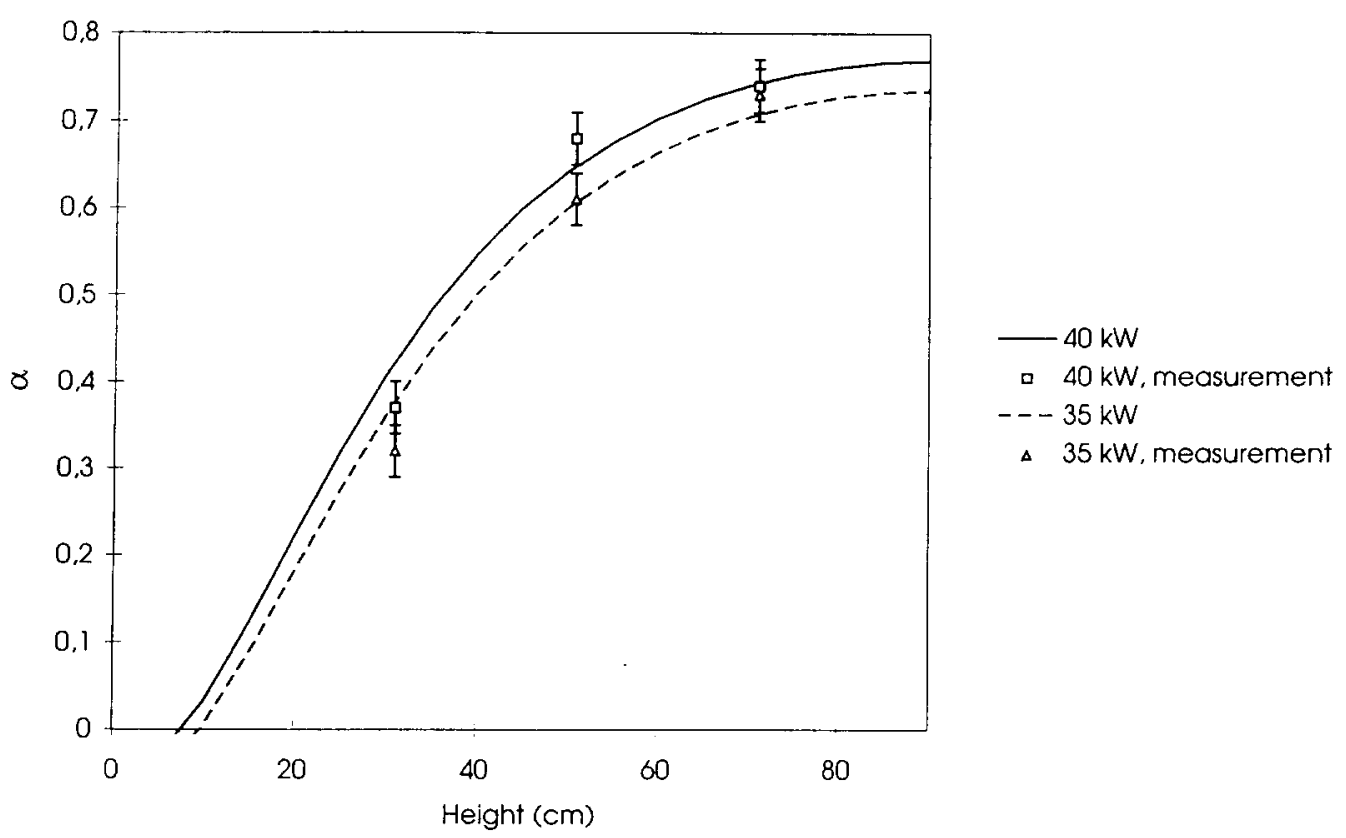

Figure 2. Measured and calculated void fraction as a function of the height in experiments with 35 and $40 \mathrm{~kW}$.

for 40 and $35 \mathrm{~kW}$ heating powers. In the calculations, a homogeneous equilibrium flow model is used with no slip between liquid and gaseous phases. The measured void fraction values match the homogeneous flow model at 51 and $71 \mathrm{~cm}$ at both heating power levels. This suggests that the flow is in thermal equilibrium at these heights. At $31 \mathrm{~cm}$, however, the void fraction is somewhat lower than the value predicted by the homogeneous model. Furthermore, temperature measurements given in table 1 indicate that the bulk of the liquid is subcooled at $31 \mathrm{~cm}$ in the experiment with $35 \mathrm{~kW}$.

The deviation from the homogeneous equilibrium model at $31 \mathrm{~cm}$ can be explained by the slip in the lower part of the fuel assembly. The void fraction as a function of the height shows a rapid increase at the lower section of the rod bundle. This yields a large acceleration of the flow. At lower axial levels, relatively little mixing takes place between the regions near the rods and the bulk of the (subcooled) liquid in central positions, respectively. The bulk of the liquid cannot follow this large acceleration which means that the vapor accelerates instead. This results in high slip factor and lower void fraction. At higher elevation, the void fraction does not increase as rapidly and there is more mixing because of the higher turbulence in the bulk boiling. The liquid catches up with the vapor and the slip factor approaches 1 .

The above results indicate that the bulk boiling boundary passes the height of $31 \mathrm{~cm}$ when the power is reduced from 40 to $35 \mathrm{~kW}$. In the upper part of the assembly bulk boiling occurs with large void fractions. At lower positions, single-phase flow and subcooled boiling takes place. In the case of subcooled boiling, bubbles stick to the rods and to the walls. Visual observations support this conclusion. In figure 3(a), (b), pictures taken through the viewing port at $31 \mathrm{~cm}$ are liquid at different heights

\begin{tabular}{lcc}
\hline \multirow{2}{*}{$\begin{array}{l}\text { Position } \\
\mathrm{cm})\end{array}$} & \multicolumn{2}{c}{ Subcooling $(\mathrm{C})$} \\
\cline { 2 - 3 } & $40 \mathrm{~kW}$ & $35 \mathrm{~kW}$ \\
\hline 71 & 0.0 & 0.0 \\
51 & 0.0 & 0.0 \\
31 & 0.0 & 0.5 \\
2 & 2.0 & 2.0 \\
\hline
\end{tabular}




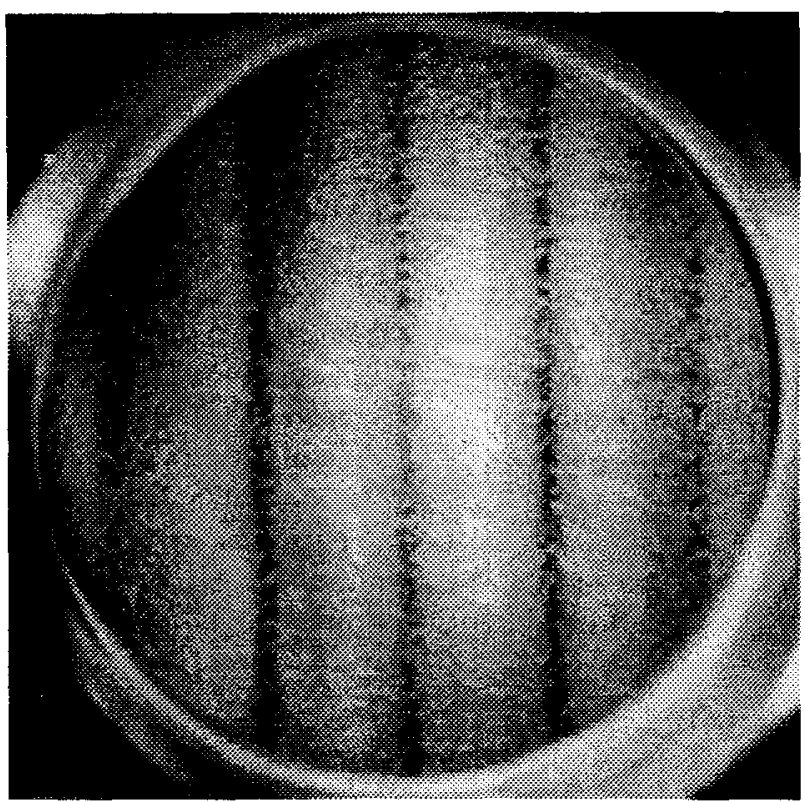

(a)

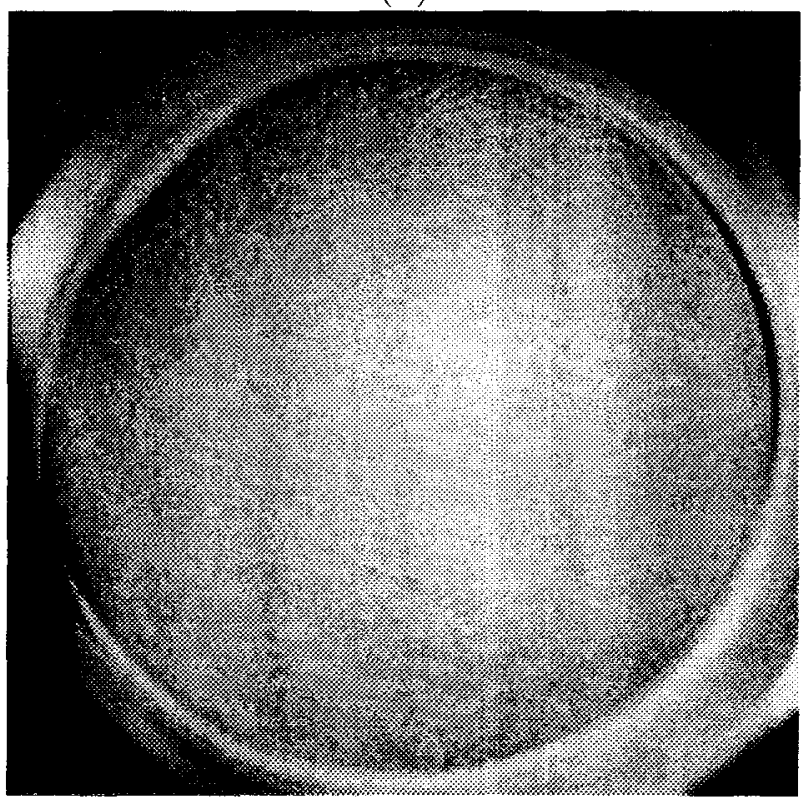

(b)

Figure 3. Boiling patterns at 35 and $40 \mathrm{~kW}$ heating powers; photographs taken through viewing port at height $31 \mathrm{~cm}$.

shown. At the lower power $(35 \mathrm{~kW})$, bubbles are concentrated near the fuel rods, indicating that the bulk of the liquid is still subcooled at this height. At $40 \mathrm{~kW}$, the liquid is saturated and bubbles are present through the whole cross-section of the channel. It is difficult to see in figure $3(\mathrm{~b})$, but bubbles coalesce in the central regions of the bundle which results in churn-turbulent two-phase flow at the given thermal-hydraulic conditions.

In the following section, the changing character of the boiling process is studied based on the statistical analysis of the thermocouple signals. 


\section{ANALYSIS OF THERMOCOUPLE SIGNALS}

\subsection{Results by spectrum and momentum analyses}

Analysis of fluctuating phenomena in two-phase flows was performed by using probability density functions (pdfs) and their moments, as well as their power spectral densities (PSD). The typical behavior of the fluctuating component of the thermocouple signals is shown in figures 4(a)-(d) and 5(a)-(d) for experiments with 40 and $35 \mathrm{~kW}$, respectively. The sampling time was $30 \mathrm{~ms}$. Strong periodic oscillations can be seen in figures 4(a), (b) and 5(a), (b), while the intensity of the oscillations ceases toward the bottom of the channel; see figures 4(c), (d) and 5(c), (d).

Changes in the character of the signals can be described quantitatively by evaluating the moments of the pdf. The variance is the second moment of the pdf. The third moment (skewness) and forth moment (kurtosis) measure the deviation of a pdf from the Gaussian pattern. Skewness measures the symmetry of the pdf, while the kurtosis indicates its peakedness. For a Gaussian distribution the skewness is zero and the kurtosis is 3 . Results obtained by momentum analysis are shown in table 2. The variance increases with the intensification of the boiling at higher elevation. The third

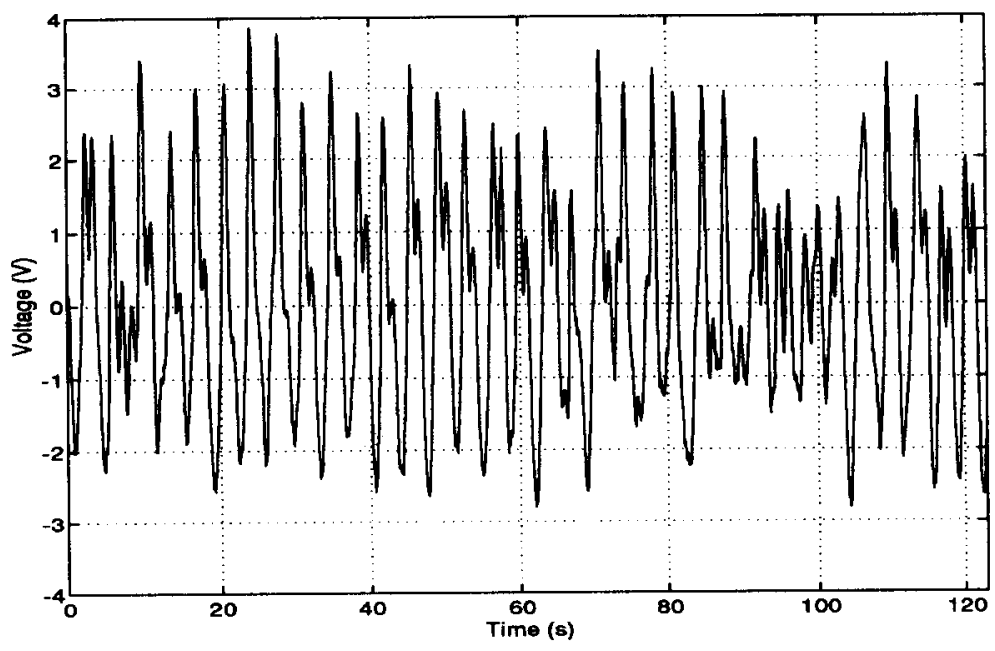

(a)

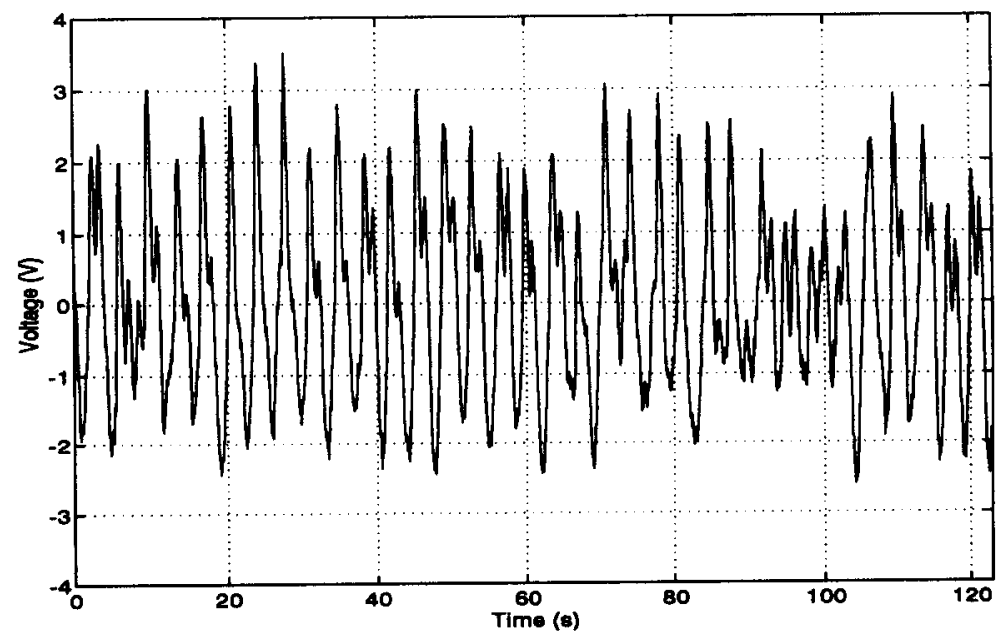

(b)

Figure 4(a, b). 


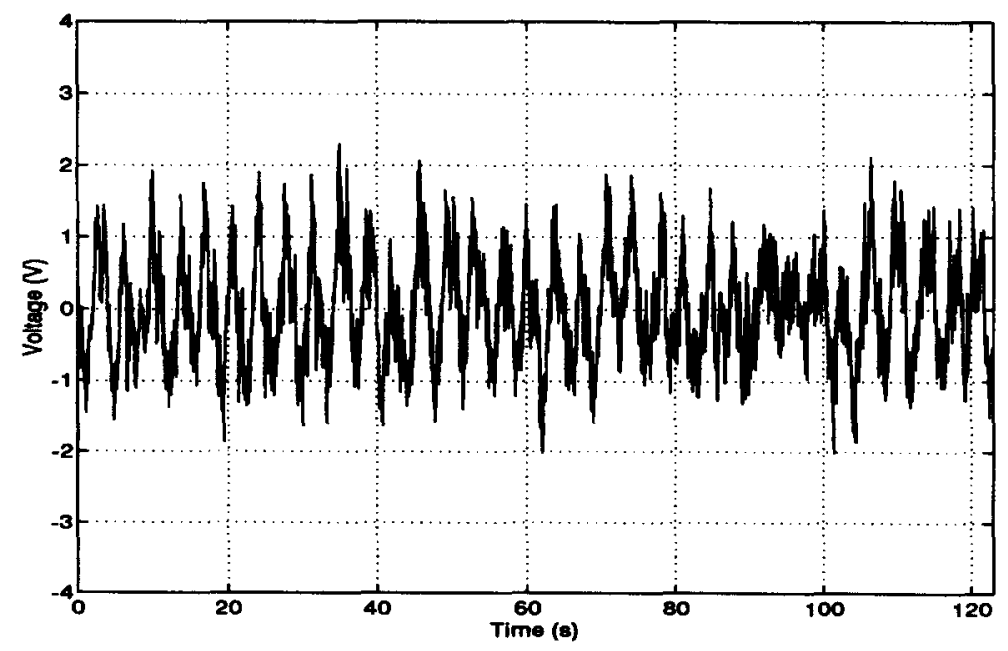

(c)

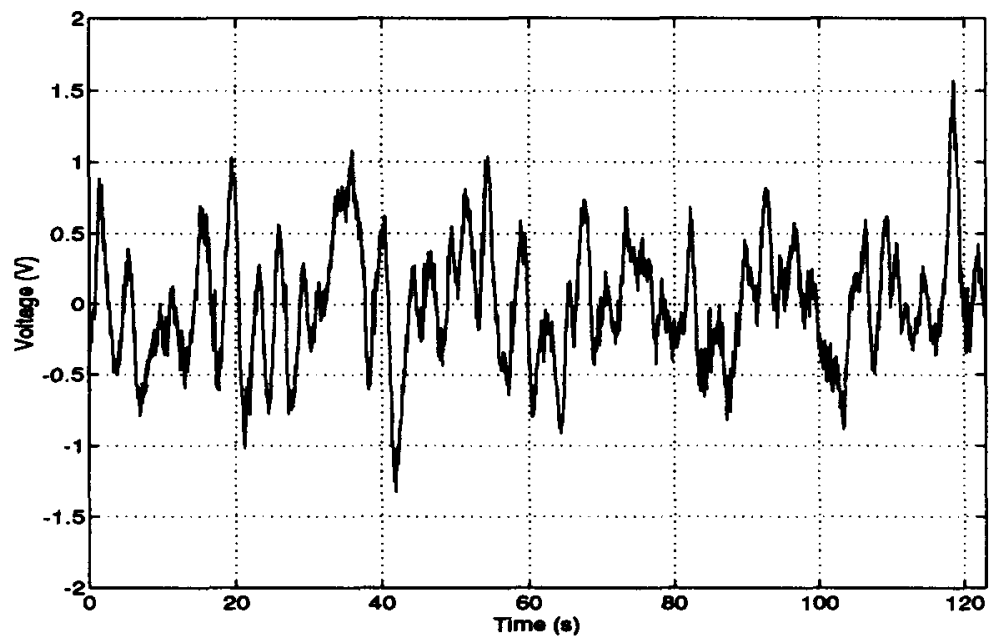

(d)

Figure 4(c, d).

Figure 4. Fluctuating part of the thermocouple signals at heating power $40 \mathrm{~kW}$; (a) $71 \mathrm{~cm}$, (b) $51 \mathrm{~cm}$, (c) $31 \mathrm{~cm}$, (d) $2 \mathrm{~cm}$.

moment more and more deviates from zero at the higher regions of the channel. The fourth moment of the pdf is close to 3 at the lower part of the channel and it decreases at the central and top positions.

Power spectral densities of the temperature signals have been calculated by an FFT algorithm. In figure 6(a)-(d), PSD functions of the measured time series are shown. Dashed and solid lines show the experiments with 35 and $40 \mathrm{~kW}$ heating power, respectively. The spectrum has a broad-band character at the bottom of the channel. There are clear peaks in the spectra measured at higher axial positions, indicating the presence of periodic oscillations in the two-phase flow. PSDs in the upper part of the channel have high peaks in the low-frequency region, at about $0.3 \mathrm{~Hz}$. The peaks are less pronounced in the case of the reduced heating power level of $35 \mathrm{~kW}$. The shape of the PSDs, however, are rather uncertain due to the relatively small number of data points. 20 data blocks have been averaged in the evaluation of the PSDs, which gives a total data duration of about $5 \mathrm{~min}$ for each spectrum. 
The results can be interpreted as follows. In the upper part of the channel, bulk boiling with churn-turbulent flow regime takes place which is a bimodal flow pattern. Oscillations in the bimodal flow are detected by the low kurtosis value, large variance, and by the peaks in the PSDs. These results are in full accordance with Vince \& Lahey (1982). At low and medium elevation, the oscillating character of the two-phase flow ceases and the flow becomes unimodal. At the bottom of the channel, the variance of the fluctuations is about 10 times smaller than at the top, the skewness is close to zero and the kurtosis approaches 3 . These observation indicate the transition to unimodal flows at lower positions.

\subsection{Result by fractal analysis}

The measured time signals are analysed by fractal methods based on [1]. The mean partial lengths of the time series are depicted in figure $7(\mathrm{a})-(\mathrm{d})$, where solid lines indicate the experiment with $35 \mathrm{~kW}$ and dashed lines stand for $40 \mathrm{~kW}$. Changes in the boiling state of the coolant manifest themselves in varying fractal properties of the signals. The $\langle L(k)\rangle$ curves in figure 7 (a)-(d) show

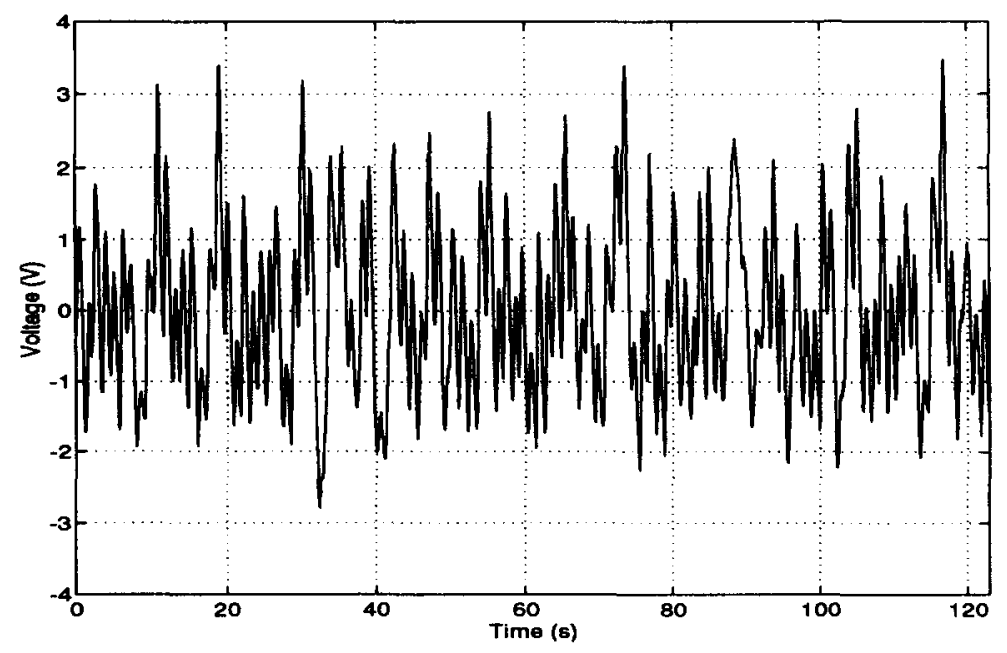

(a)

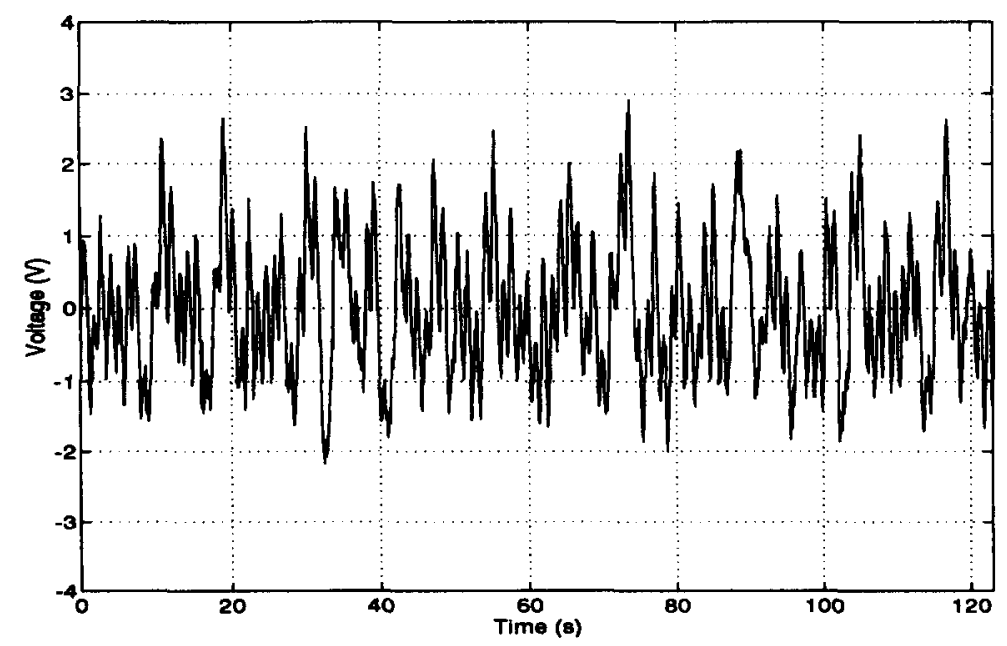

(b)

Figure 5(a, b). 


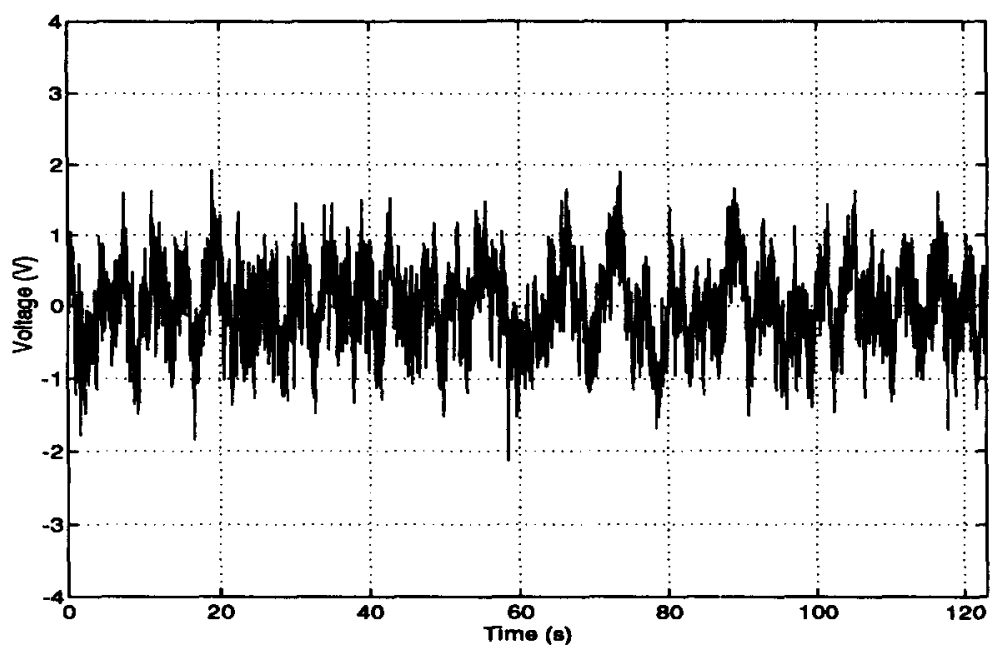

(c)

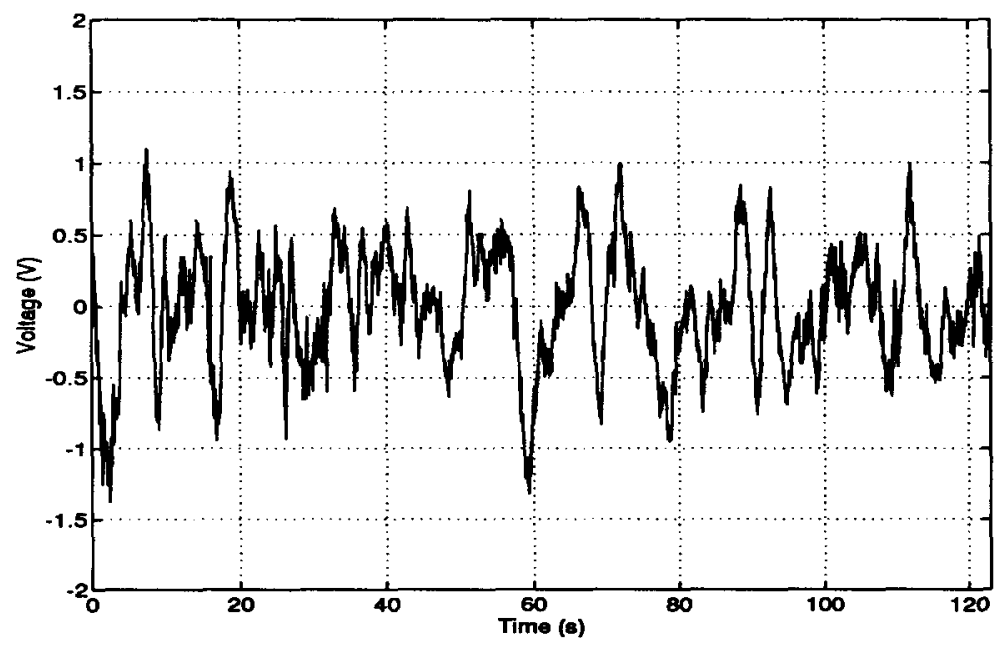

(d)

Figure 5(c, d).

Figure 5. Fluctuating part of the thermocouple signals at heating power $35 \mathrm{~kW}$; (a) $71 \mathrm{~cm}$, (b) $51 \mathrm{~cm}$, (c) $31 \mathrm{~cm}$, (d) $2 \mathrm{~cm}$.

a decreasing tendency as $k$ increases. Detailed analysis reveals certain nonlinearities in the slope of these curves.

Two major effects will be analysed. The first one is the bi-fractal behavior, i.e. the presence of a breaking point in the $\log (\langle L(k)\rangle)$ curve at intermediate $k$ values. In the case of higher heating power, the $\log (\langle L(k)\rangle)$ curve deviates more from the linear behavior; see figure $7(a)-(d)$. The

Table 2. Moments of the probability density functions

\begin{tabular}{lccccccc}
\hline \multirow{2}{*}{$\begin{array}{l}\text { Vertical } \\
\text { position }\end{array}$} & \multicolumn{3}{c}{$40 \mathrm{~kW}$} & & \multicolumn{3}{c}{$35 \mathrm{~kW}$} \\
\cline { 2 - 4 } \cline { 6 - 8 } & 2 & 3 & 4 & & 2 & 3 & 4 \\
\hline $72 \mathrm{~cm}$ & 2.15 & 0.27 & 2.32 & & 1.36 & 0.46 & 2.61 \\
$51 \mathrm{~cm}$ & 1.67 & 0.21 & 2.27 & & 0.83 & 0.43 & 2.67 \\
$31 \mathrm{~cm}$ & 0.52 & 0.19 & 2.52 & 0.34 & 0.04 & 2.87 \\
$2 \mathrm{~cm}$ & 0.16 & 0.19 & 3.10 & 0.17 & -0.04 & 2.84 \\
\hline
\end{tabular}




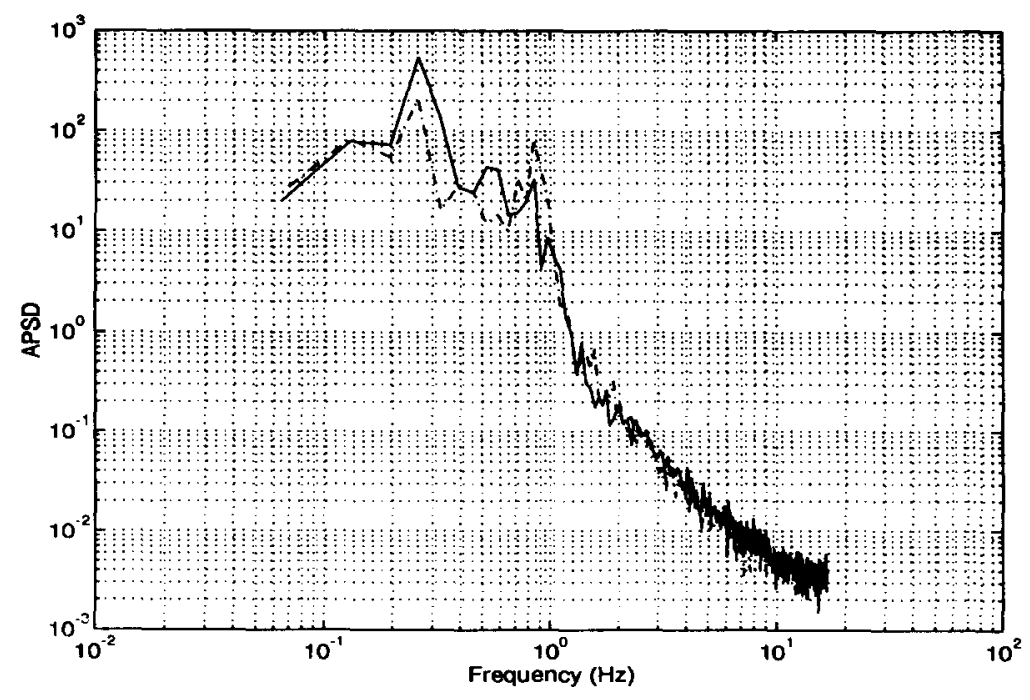

(a)

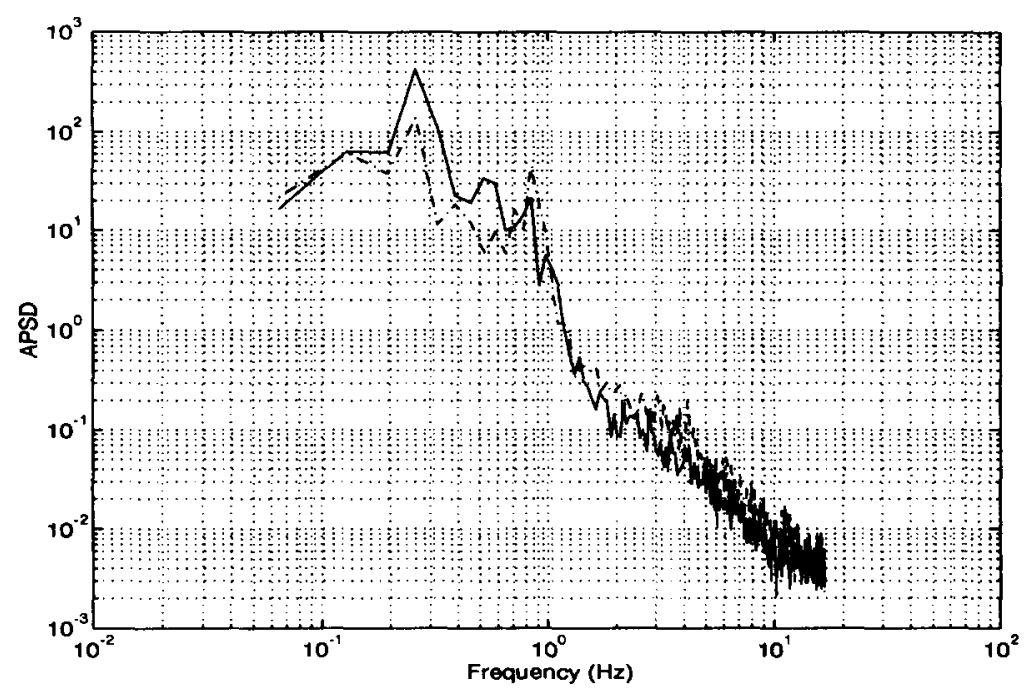

(b)

Figure 6(a,b).

second effect is related to the error of the linear fit of the fractal curves. Visual inspection of the curves in figure $7($ a) $-(d)$ indicates that the fractal curves have more intensive fluctuations at higher heating powers and higher detector positions.

In order to describe these observations quantitatively, the fractal dimension value and its error have been estimated based on the method described in section 2 . The calculations have been conducted over the $k$ range from 5 to 512, and over sub-ranges 5-53 and 53-512. The fractal curves have linear behavior starting from $k=1$. In the fitting algorithm, however, the smallest $k$ values have been omitted from the fitting in order to exclude high-frequency distortions to the signals. Examples of bi-fractal fit are shown in figure 8(a), (b) for detectors at positions 71 and $2 \mathrm{~cm}$, respectively.

The numerical evaluations of the fractal dimension are summarized in table 3 . At the higher $k$-range, the fractal dimension has a rather constant value close to 2 , independent of the 
thermocouple position. Note that the fractal dimension value is 2 for a Gaussian random signal. At small $k$ values, the fractal dimension varies between 1.3 and 1.8. $D$ over small $k$ values has a minimum value at the bottom of the channel. The difference between the fractal dimension at small and large $k$ values, respectively, is significantly reduced at $31 \mathrm{~cm}$ at both power levels. In order to estimate the significance of the observed changes in the fractal dimension, the error of the linear fit of the $\log (k)$ vs $\log (\langle L(k)\rangle)$ curve has been determined. The fitting error of the determined fractal dimension is denoted by $\sigma_{D}$ and its values are given in table 4 based on the sample standard deviation of the linear fit of the fractal curves.

$\sigma_{D}$ over the $k$ range $[5,512]$ is a good indication of the importance of the break observed in figure $7(\mathrm{a})-(\mathrm{d}) . \sigma_{D}$ is larger in the experiments with higher heating power. Its value is essentially constant

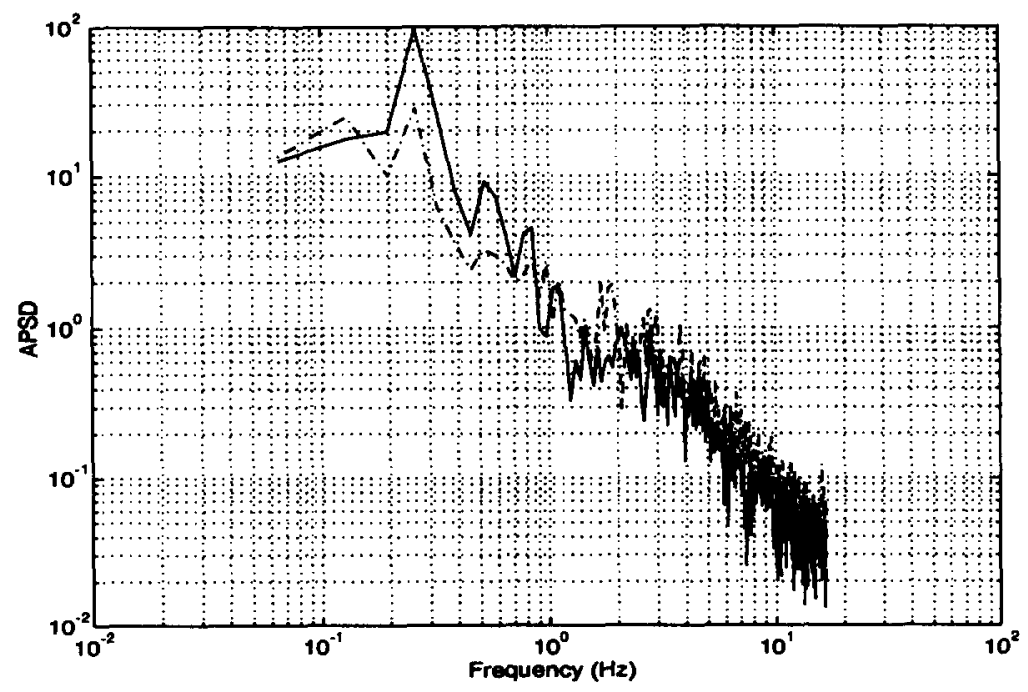

(c)

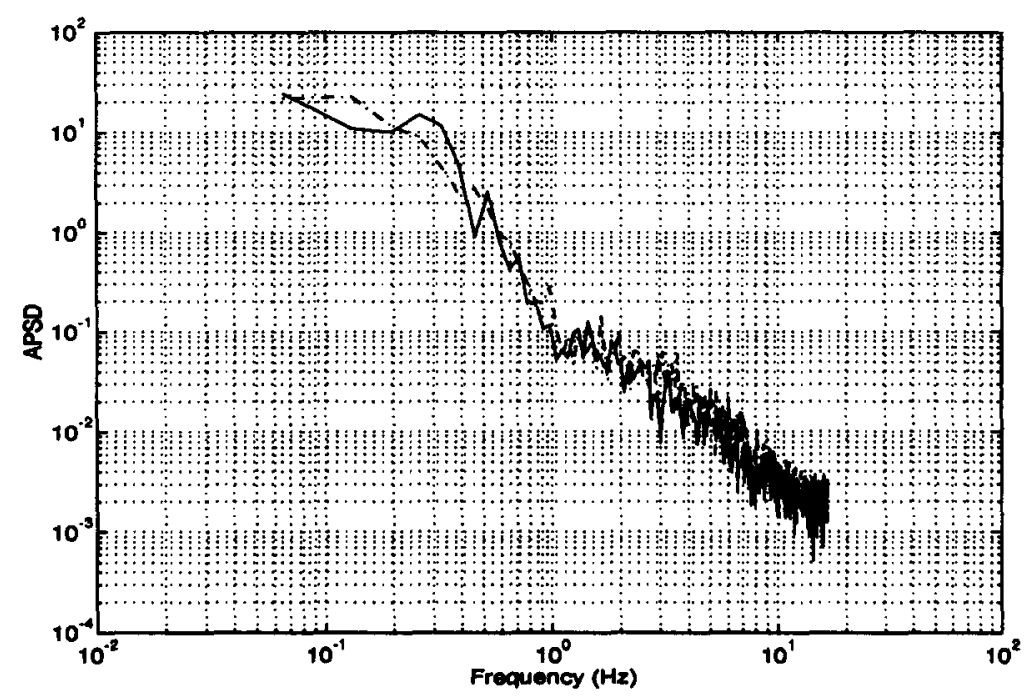

(d)

Figure 6(c, d),

Figure 6. Power spectra of thermocouple signals; dashed line- $-35 \mathrm{~kW}$, solid line-40 kW; (a) $71 \mathrm{~cm}$, (b) $51 \mathrm{~cm}$, (c) $31 \mathrm{~cm}$, (d) $2 \mathrm{~cm}$. 


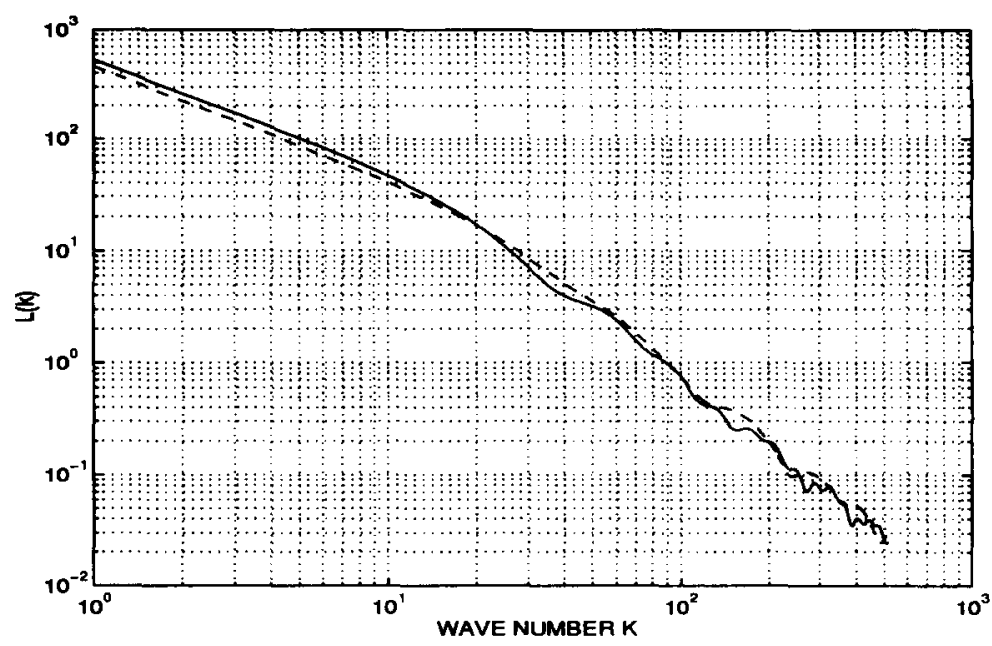

(a)

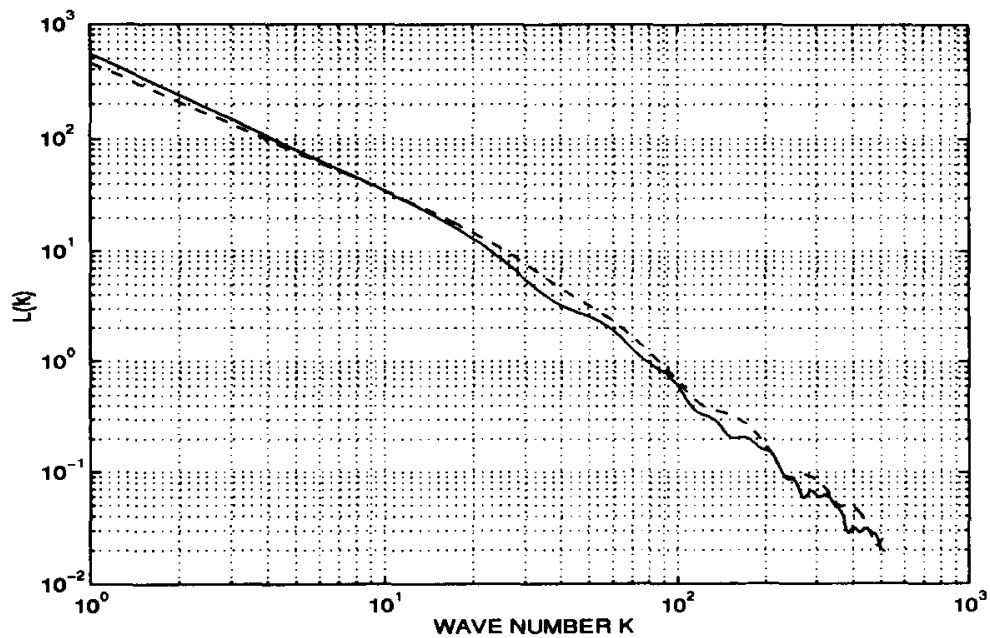

(b)

Figure $7(a, b)$.

along the channel except for a decrease at the detector position $31 \mathrm{~cm}$. Over the $k$-range [5, 53], $\sigma_{D}$ is small at 2 and $31 \mathrm{~cm}$, but it jumps to a 3-4-times larger value at higher detector positions. Similar jumps are observed over the $k$-range of $[53,512]$ in the experiment with $35 \mathrm{~kW}$. The increase of the error along the channel is more continuous in experiment with $40 \mathrm{~kW}$, when the error level (0.073) has an intermediate value between the minimum (0.035) and maximum (0.115). The interpretation of these results is given in the next section.

\section{DISCUSSION OF THE RESULTS BY FRACTAL METHODS}

Two typical time scales can be distinguished in the measured signals with the different dispersions of fractal dimension $D$. The value of $D$ was around 2 over the $k$-range [53,512] for all detector positions. $D$ is smaller than 2 over the $k$-range $[5,53]$ and it varies significantly for various heating conditions and axial detector locations; see table 3. These changes, however, have no clear trend and they cannot form a basis of a reliable two-phase flow identification method. Further studies are necessary to understand better the relationship between $D$ and two-phase flow regimes. 
It can be seen in table 4 that $\sigma_{D}$ is increasing for higher detector positions over both the low and high $k$-ranges. Gamma-void measurements and model calculations show that subcooled boiling exists at the lower section of the assembly. Coalescence of small bubbles into big ones in a highly irregular way leads to churn-turbulent flow at higher positions with bulk boiling, i.e. starting from axial positions $30 \mathrm{~cm}$ to $35 \mathrm{~cm}$ in our experiments. The jump of $\sigma_{D}$ over the $k$-range $[5,53]$ clearly indicates the transition from bubbly flow to churn-turbulent flow.

$\sigma_{b}$ over range $[53,512]$ is even more sensitive to flow regime changes. It has an intermediate magnitude of 0.073 at position $31 \mathrm{~cm}$ in experiment with $40 \mathrm{~kW}$ heating, which indicates that the two-phase flow has departed from the bubbly regime. $\sigma_{D}$ at the same location in case of $35 \mathrm{~kW}$ heating has not changed significantly from the inlet value of 0.046 , which shows the presence of subcooled boiling with unimodal bubbly flow regime at that elevation. The large $\sigma_{D}$ at higher positions is due to intensive fluctuations of the $\log (k)$ vs $\log (\langle L(k)\rangle)$ curves which, in turn, are caused by periodic oscillations in the bimodal churn-turbulent flow. The fitting error of the fractal

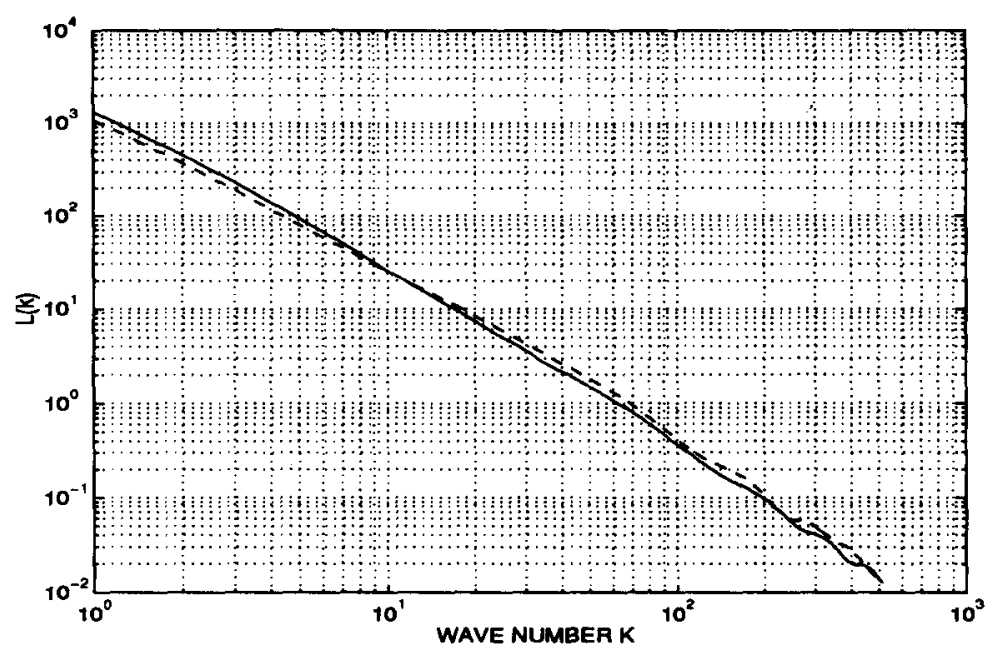

(c)

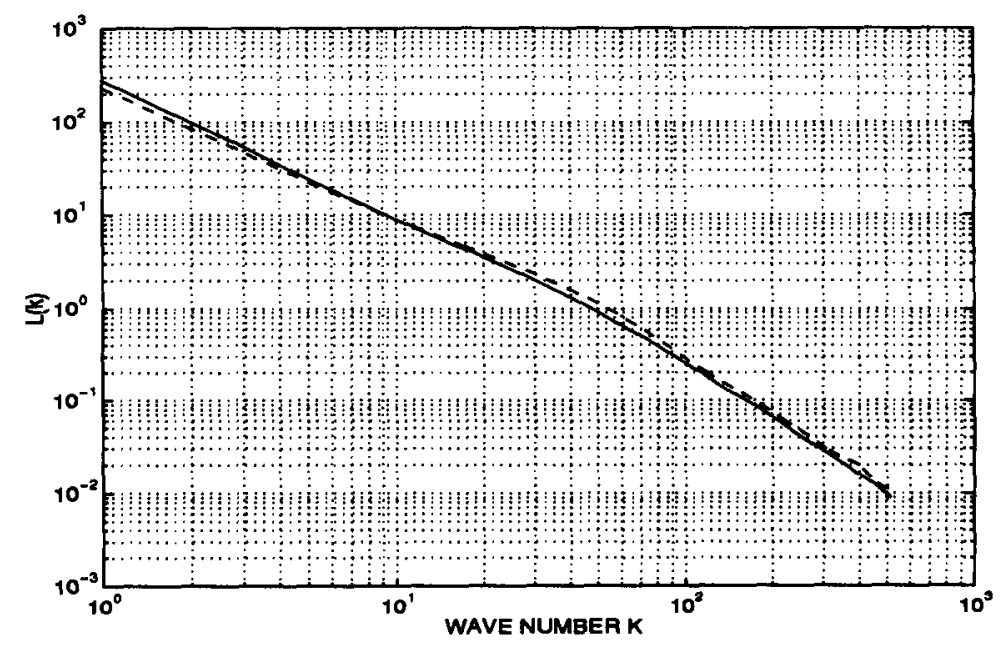

(d)

Figure $7(c, d)$.

Figure 7. $\log (k)$ vs $\log (\langle L(k)\rangle)$ plots of the fluctuating signal component, dash $-40 \mathrm{~kW}$, solid- $35 \mathrm{~kW}$; (a) $71 \mathrm{~cm}$, (b) $51 \mathrm{~cm}$, (c) $31 \mathrm{~cm}$, (d) $2 \mathrm{~cm}$. 


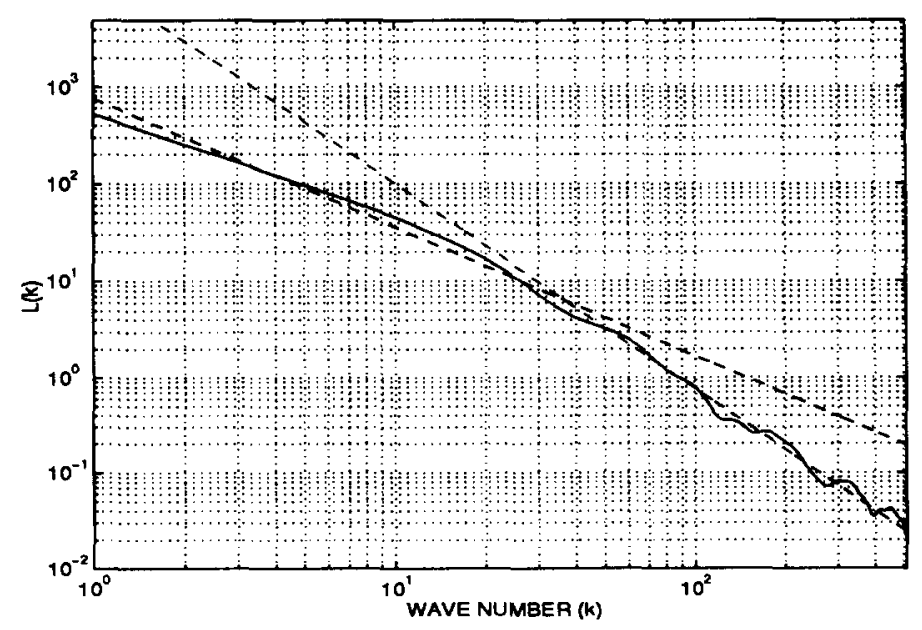

(a)

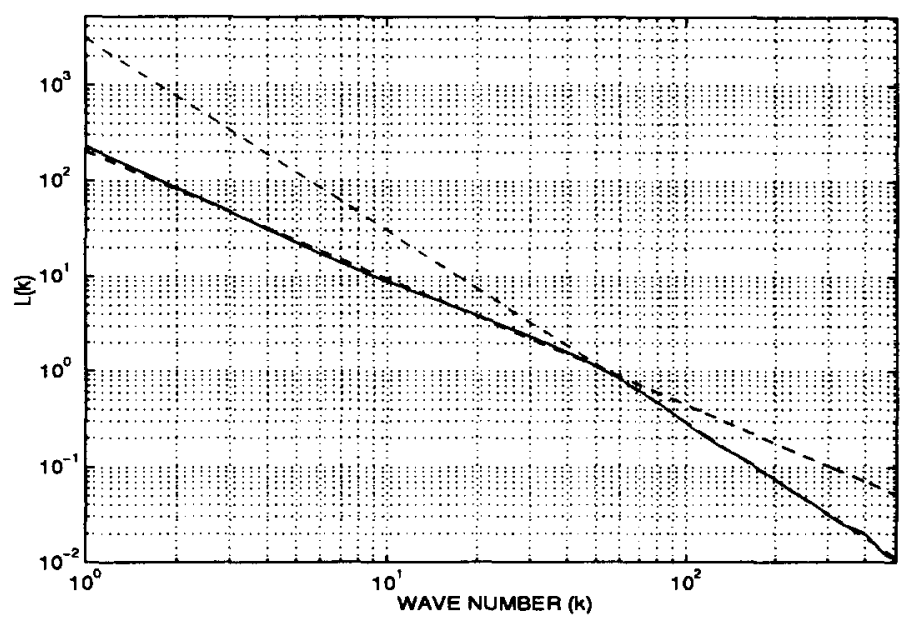

(b)

Figure 8. Bi-fractal fit of the $\log (k)$ vs $\log (\langle L(k)\rangle)$ curves in experiment with $40 \mathrm{~kW}$ heating power; (a) $71 \mathrm{~cm}$, (b) $2 \mathrm{~cm}$.

dimension over large $k$ values is proposed as a sensitive indicator of the changes in the two-phase flow regime.

The physical interpretation of the proposed flow regime indicator based on the variance of the fractal dimension is given as follows. The variance of the fractal dimension has two components: (1) statistical effects caused by the limited length of time series used in the analysis; (2) the deviation of the observed time series from the self-similar behavior over a given $k$-range; Higuchi (1988,

Table 3. Fractal dimension of the measured signals

\begin{tabular}{lccccccc}
\hline & \multicolumn{3}{c}{$40 \mathrm{~kW}$} & \multicolumn{3}{c}{$35 \mathrm{~kW}$} \\
\cline { 2 - 4 } \cline { 6 - 8 } vertical & $5 \leqslant k \leqslant 512$ & $5 \leqslant k \leqslant 53$ & $53 \leqslant k \leqslant 512$ & $5 \leqslant k \leqslant 512$ & $5 \leqslant k \leqslant 53$ & $53 \leqslant k \leqslant 512$ \\
\hline $71 \mathrm{~cm}$ & 1.79 & 1.39 & 2.07 & 1.87 & 1.59 & 2.09 \\
$51 \mathrm{~cm}$ & 1.78 & 1.38 & 2.07 & 1.86 & 1.58 & 2.09 \\
$31 \mathrm{~cm}$ & 1.85 & 1.63 & 2.05 & & 1.91 & 1.80 & 2.04 \\
$2 \mathrm{~cm}$ & 1.65 & 1.27 & 2.01 & 1.71 & 1.38 & 2.03 \\
\hline
\end{tabular}


Table 4. Standard deviation of fractal fit

\begin{tabular}{lccccccc}
\hline & \multicolumn{3}{c}{$40 \mathrm{~kW}$} & & \multicolumn{3}{c}{$35 \mathrm{~kW}$} \\
\cline { 2 - 4 } \cline { 6 - 8 } vertical & & $5 \leqslant k \leqslant 512$ & $5 \leqslant k \leqslant 53$ & $53 \leqslant k \leqslant 512$ & $5 \leqslant k \leqslant 512$ & $5 \leqslant k \leqslant 53$ & $53 \leqslant k \leqslant 512$ \\
\hline $71 \mathrm{~cm}$ & 0.111 & 0.084 & 0.114 & & 0.074 & 0.123 & 0.102 \\
$51 \mathrm{~cm}$ & 0.113 & 0.079 & 0.115 & & 0.076 & 0.104 & 0.102 \\
$31 \mathrm{~cm}$ & 0.073 & 0.016 & 0.073 & & 0.040 & 0.019 & 0.049 \\
$2 \mathrm{~cm}$ & 0.112 & 0.024 & 0.035 & & 0.091 & 0.027 & 0.046 \\
\hline
\end{tabular}

1990). A comprehensive study of the above two effects is given by Sakuma (1996). In the present study the parameters of the evaluation of the fractal dimension are fixed, therefore, the observed effects are caused by changes in the self-similarity of the measured signals.

A linear fractal curve over a given $k$-range indicates complete self-similarity. Previous studies showed that self-similarity is a characteristic property of turbulence; see, e.g. Marvasti et al. (1995). The increasing variance of the fractal dimension means deviation from self-similarity. The physical reason of the changing self-similarity in the two-phase flow measurements is the emergence of certain dominant frequencies. Changes in the frequency content of the signal can be monitored by spectrum analysis as well. However, fractal-based methods have a clear advantage compared to spectrum analysis, due to the non-stationary and intermittent nature of turbulent fluctuations in two-phase flows. In the next section, the comparison of spectral and fractal methods is given.

There is a close relationship between the fractal dimension and the shape of the power spectral densities in the case of fractal time series. It can be shown that the following relationship holds between the slope $\alpha$ of the PSD in the $\log -\log$ axis and the fractal dimension $D$ of a fractal signal (Higuchi, 1990):

$$
\alpha=5-2 D .
$$

For a bi-fractal signal, the PSD has two frequency regions with constant slopes and the above relationship is valid for $\alpha$ and $D$ values inferred for each of the frequency- and $k$-ranges.

Figure 6(a), (b) show that the definition of the frequency range used for the evaluation of the PSD slope is not easy due to large oscillations in the spectra. The fractal curves in figure 7(a), (b), however, are relatively smooth and the fitting of its segments is an easier task. This result shows the advantage of the fractal method compared to spectrum analysis in our experiments.

It is also clear from the definition of the fractal dimension based on [1] that $D$ is invariant to a constant multiplication factor applied to the signal. If the changes in the signal absolute magnitude are of importance to the description of the physical process, complementary methods are required in addition to fractal analysis. This is clearly the case in two-phase flow regime monitoring, when the variance of the pdf is an important indicator of flow regime changes; see Vince \& Lahey (1982). This result shows that fractal methods should be used in combination with other methods in order to achieve an effective two-phase flow characterization and identification method.

\section{CONCLUSIONS}

In the paper, results of two-phase flow identification experiments performed at the SIDAS boiling loop are introduced. At the lower section of the rod bundle, single-phase flow and subcooled boiling took place, while there is bulk boiling with churn-turbulent flow regime at higher axial elevation. The major results of boiling two-phase flow identification can be summarized as follows:

(1) Based on the analysis of moments of probability density function of various thermocouples in the coolant as well as using spectrum analysis, bubbly and churn-turbulent two-phase flow regimes have been identified in the boiling channel. Visual observations and gamma-void measurements supported the identification. These results are in accordance with previous results by Vince \& Lahey (1982) and Dukler \& Taitel (1986).

(2) By applying Higuchi's fractal dimension evaluation algorithm, a bi-fractal behavior is found in the measured signals. The fractal dimension $D$ is approximately 2 over the range of large wave numbers $k$. This $D$ value is quite independent of the actual boiling regime and of the 
axial position of the detectors. The fractal dimension over the small wave number range, on the other hand, is a function of the two-phase flow regime but no clear correlation could be established between the fractal dimension value and the two-phase flow regime.

(3) In the present study, the standard deviation of the linear fit of the $\log (k)$ vs $\log (\langle L(k)\rangle)$ curve over the range of large $k$ numbers is proposed as a sensitive indicator of the transition between flow regimes. This standard deviation is $2-4$ times larger in churn flow than in unimodal bubbly flow. The increased standard deviation is attributed to the diminishing self-similarity in the signal caused by the emerging periodic component in bi-modal flows. The main advantage of the fractal method compared to spectrum analysis is that it can clearly identify intermittent oscillations in non-stationary two-phase flows. These results support the feasibility of flow pattern identification method based on fractal analysis.

Further studies are going on to demonstrate the advantages of fractal methods in the interpretation of non-stationary/transient data. Model calculations and two-phase flow simulations can contribute to the understanding of the nature of complicated processes in the highly irregular, boiling two-phase flows.

Acknowledgements - This work has been completed in the framework of the research cooperation between Department of Nuclear Engineering, Tohoku University, Sendai, and Department of Reactor Physics, IRI, Delft University of Technology, Delft. The experiments have been performed at the SIDAS facility during the visit of two of the authors (R.K. and M.S.) at IRI. The authors wish to thank the staff of IRI for their assistance during the experiments and to Dr Valerie Kozma-Blazsik for editing the manuscript.

\section{REFERENCES}

Baba, N., Yamashita, Y. \& Shiraishi, Y. 1991 Classification of flow patterns in two-phase flow by neural networks, Proc. IEEE Int. Conf. Neural. Netw. 2, 2617-2620.

Djainal, D. D., Sakuma, M., Kozma, R. \& Kitamura, M. 1995 Characterization of two-phase flow patterns using fractal techniques. Proc. 2nd Int. Conf. Multiph. Flow, Kyoto' 95, Vol. 3, FT1. pp. 31-36.

Dukler, A. E. \& Taitel, Y. 1986 Flow pattern transitions in gas-liquid systems: measurement and modeling. Multiphase Sci. and Technol. 2, 1-94.

Franca, F., Acikgoz, M., Lahey, R. T. Jr \& Clausse, A. 1991 The use of fractal techniques for flow regime identification. Int. J. Multiphase Flow 17, 545-552.

Graaf, van de R., van der Hagen, T. H. J. J. \& Mudde, R. F. 1994 Scaling laws and design aspects of a natural-circulation-cooled simulated boiling water reactor fuel assembly. Nucl. Technol. 105, 190-200.

Higuchi, T. 1988 Approach to an irregular time series on the basis of the fractal theory. Physica $D$ 31, 277-283.

Higuchi, T. 1990 Relationship between fractal dimension and power law index for a time series: a numerical investigation. Physica D 46, 254-264.

Kozma, R., van Dam, H. \& Hoogenboom, J. E. 1992 Identification of flow patterns by neutron noise analysis during actual coolant boiling in thin rectangular channels. Nucl. Technol. 100, 97-110.

Mandelbrot B. B. 1982 The Fractal Geometry of Nature. Freeman, New York.

Marvasti, M. A. \& Strahle, W. C. 1995 Fractal geometry analysis of turbulent data. Signal Processing 41, 191-201.

Saether, G., Bendiksen, K., Müller, J. \& Fröland, E. 1990 The fractal statistics of liquid slug lengths. Int. J. Multiphase Flow 16, 1116-1126.

Sakuma, M., Kozma, R. \& Kitamura, M. 1996 Detection and characterization of anomalies by applying methods of fractal analysis. Nucl. Technol. 113, 86-99.

Schepers, H. E., van Beek, H. G. M. \& Bassingthwaighte, J. B. 1992 Four methods to estimate the fractal dimension from self-affine signals. IEEE Engng in Medicine and Biology 11, 57-64.

Vince, M. A. \& Lahey, R. T. Jr 1982 On the development of an objective flow regime indicator. Int. J. Multiphase Flow 8, 93-124. 